\title{
Manifestaciones clínicas y resultados oncológicos del cáncer renal en un hospital del norte de México
}

\section{Clinical presentation and oncologic results of kidney cancer at a hospital in Northern Mexico}

\author{
Jaime Uscanga-Yépez, ${ }^{1}$ Ana Martínez-González, ${ }^{2}$ Karen Segovia-Sandoval, ${ }^{2}$ Eduardo Barrera-Juárez, ${ }^{3}$ Roberto \\ Gonzalez-Oyervides ${ }^{4}$
}

\section{Resumen}

OBJETIVO: Reportar la experiencia en el tratamiento quirúrgico de pacientes con carcinoma de células renales y las características histopatológicas de éstas en una institución del norte de México.

MATERIALES Y MÉTODOS: Estudio transversal y descriptivo efectuado mediante la revisión de los expedientes de pacientes intervenidos de tumor renal. La decisión de realizar la nefrectomía radical o parcial se estableció según el puntaje de la nefrometría obtenida por tomografía de abdomen contrastada y el criterio del cirujano. Las comorbilidades evaluadas fueron: hipertensión arterial sistémica, diabetes tipo 2, hematuria macroscópica, dolor en el flanco y aumento de volumen en el hipocondrio. También se registró la tasa de filtración glomerular (TFG) antes de la cirugía y en los primeros 15 días posquirúrgicos. La tasa de filtración glomerular se calculó con la fórmula MDRD (Modification of Diet in Renal Disease). Las variables se analizaron con medidas de tendencia central y la supervivencia con las curvas de Kaplan-Meier.

RESULTADOS: Se registraron 89 casos. El 74\% manifestó algún síntoma, principalmente hematuria y dolor en el flanco. Predominó la nefrectomía radical, con una media de tamaño tumoral de $6.7 \mathrm{~cm}$. El 84\% de los casos se clasificó en estadios T1b al T4; la supervivencia cáncer específica estimada a 5 años fue de $90 \%$ y supervivencia libre de recurrencia de $83 \%$. El tipo de cirugía no influyó estadísticamente en la función renal posoperatoria $(p=0.33)$.

CONCLUSIÓN: Las características histopatológicas, el tratamiento y la supervivencia de pacientes con cáncer renal son similares a las reportadas en otros países.

PALABRAS CLAVE: Cáncer renal; nefrectomía; pronóstico; supervivencia.

\section{Abstract}

OBJECTIVE: To report our experience with the surgical treatment and histopathologic characteristics of patients with renal cell carcinoma at a hospital in Northern Mexico. MATERIALS AND METHODS: A descriptive, cross-sectional study was conducted through the review of the case records of patients operated on for kidney tumor. Radical or partial nephrectomy was decided upon, according to the nephrometry score from contrastenhanced abdominal tomography and the criterion of the surgeon. The comorbidities evaluated were: high blood pressure, type 2 diabetes mellitus, gross hematuria, flank pain, and increased volume in the hypochondrium. Glomerular filtration rate was registered before surgery and 15 days after the procedure and was calculated using the Modification of Diet in Renal Disease (MDRD) formula. Variables were analyzed using measures of central tendency and survival was assessed through the KaplanMeier curves.

RESULTS: Eighty-nine cases were registered. Symptoms presented in $74 \%$ the most common of which were hematuria and flank pain. Radical nephrectomy predominated, and the mean pathologic tumor size was $6.7 \mathrm{~cm}$. A total of $84 \%$ of the cases had stage

\footnotetext{
${ }^{1}$ Urólogo, miembro del cuerpo médico, The American British Cowdray Medical Center, I.A.P.

${ }^{2}$ Departamento de Urología.

${ }^{3}$ Director académico de la especialidad en Urología.

${ }^{4}$ Profesor de la especialidad en Urología.
}

Hospital Metropolitano Dr. Bernardo Sepúlveda, Nuevo León; Escuela Nacional de Medicina, Instituto Tecnológico de Estudios Superiores de Monterrey, Nuevo León.

Recibido: enero 2018

Aceptado: mayo 2018

Correspondencia Jaime Uscanga Yépez j.uscanga.y@gmail.com

Este artículo debe citarse como Uscanga-Yépez J, Martínez-González A, Segovia-Sandoval K, Barrera-Juárez $\mathrm{E}$, González-Oyervides R. Manifestaciones clínicas y resultados oncológicos de cáncer renal en un hospital del norte de México. Rev Mex Urol. 2018 mayojunio;78(3):176-182.

DOI:https://doi.org/10.24245/revmexurol.v78i3.1929 
T1b to T4 disease. Estimated 5-year cancer-specific survival for all stages was $90 \%$ and recurrence-free survival for organ-confined stages was $83.1 \%$. The type of surgery had no statistical influence on postoperative kidney function $(p=0.33)$.

CONCLUSION: The histopathologic characteristics, treatment, and survival in patients with kidney cancer were similar to those reported in other countries.

KEYWORDS: Kidney cancer; Nephrectomy; Outcome; Survival.

\section{ANTECEDENTES}

El cáncer renal representa $2-3 \%$ de todas las neoplasias. ${ }^{1}$ Su incidencia se ha incrementado en las últimas décadas, además de la detección de tumores incidentales mediante estudios de imagen con mayor sensibilidad. ${ }^{2}$ En 2012 se estimaron 143,000 fallecimientos en todo el mundo por esta enfermedad, incluso sugirió la décima causa de cáncer en hombres y mujeres. En 2017 se diagnosticaron 63,990 nuevos casos en Estados Unidos. ${ }^{4}$ El cáncer de células renales puede manifestarse como una lesión indolente o evolucionar a una neoplasia muy agresiva. ${ }^{5}$ La forma de manifestación influye en la supervivencia a la enfermedad, incluso supone tasas más altas en pacientes asintomáticos, con diagnóstico fortuito. ${ }^{6}$ Entre las diversas opciones de tratamiento se incluyen: vigilancia activa, termoablación y cirugía. La nefrectomía parcial representa una opción para masas renales de cualquier tamaño, clásicamente tumores de 4 $\mathrm{cm}$ o menores; no obstante, se ha demostrado segura en tumores menores de $7 \mathrm{~cm} .{ }^{1}$ La media de supervivencia en pacientes con cáncer de células renales a 6 meses se estima en $76 \%$ para todos los grupos de riesgo. ${ }^{7}$

Los países industrializados han experimentado un aumento en la incidencia de tumores detectados de manera fortuita desde la década pasada, incluso en $59 \%$ de los casos. ${ }^{8}$ De igual manera, la proporción de estadios clínicos T1-T2 se ha incrementado de 49 a $74 \%$, mientras que la de pacientes con enfermedad metastásica M1 ha disminuido de 20 a $10 \% .{ }^{9}$ La supervivencia a 5 años para cáncer localizado se incrementó de 88\% (entre 1992 y 1995) a 93\% (de 2006 a 2012) y para enfermedad avanzada de 7\% (de 1992 a 1995) a $12 \%$ (de 2006 a 2012). ${ }^{7,10}$

En la actualidad existen diversos estudios que definen las principales variables de carcinoma de células renales en todo el mundo; sin embargo, en México y América Latina estos estudios son limitados y evidencian un notable subregistro de la incidencia, prevalencia y mortalidad. Como consecuencia es difícil establecer comparaciones y predicciones del carcinoma de células renales en estas regiones.

De acuerdo con lo anterior, el objetivo de este estudio es reportar la experiencia en el tratamiento quirúrgico y las características histopatológicas de pacientes con carcinoma de células renales en una institución del norte de México.

\section{MATERIALES Y MÉTODOS}

Estudio transversal y descriptivo, efectuado mediante la revisión de los expedientes de pacientes con tumores renales, atendidos en el Departamento de Urología del Hospital Metropolitano Dr. Bernardo Sepúlveda (SSA), entre los años 2011 y 2016. Se seleccionaron pacientes intervenidos de tumor renal, mayores de 18 años, con diagnóstico de tumor renal maligno. La decisión de realizar la nefrectomía radical o parcial se estableció según el puntaje de la nefrometría obtenida por tomografía de 
abdomen contrastada y el criterio del cirujano. Las comorbilidades evaluadas antes del procedimiento quirúrgico fueron: hipertensión arterial sistémica, diabetes tipo 2, dislipidemia y tabaquismo; manifestaciones como: hematuria macroscópica, dolor en el flanco y aumento del volumen en el hipocondrio. También se registró la tasa de filtración glomerular (TFG) antes de la cirugía y en los primeros 15 días posquirúrgicos. La tasa de filtración glomerular se calculó con la fórmula MDRD (Modification of Diet in Renal Disease), ${ }^{11}$ considerada normal en $60 \mathrm{~mL} /$ $\mathrm{min} / 1.73 \mathrm{~m}^{2}$ o superior. Los hallazgos patológicos evaluados fueron: tamaño tumoral según la clasificación TNM de 2009, propuesta por la AJCC (American Joint Committee on Cancer), ${ }^{1}$ grado de diferenciación celular (clasificación de Fuhrman), tipo histológico y tamaño patológico, revisados por el Departamento de Patología de la institución.

El tamaño tumoral y estadificación inicial se analizaron mediante tomografía con contraste endovenoso de tórax, abdomen y pelvis. Durante el seguimiento se solicitaron estudios de imagen: tomografía de abdomen y pelvis contrastada cada seis meses los primeros dos años y cada año a partir del tercero, hasta el quinto año. La radiografía de tórax se efectuó cada seis meses los primeros 2 años y a partir del tercero cada año, hasta completar cinco años. El seguimiento incluyó estudios metabólicos séricos como: biometría hemática, pruebas de funcionamiento hepático, calcio sérico y examen general de orina. La detección de recurrencia local, progresión y metástasis se determinaron en el seguimiento y la muerte se confirmó con el certificado de defunción.

Para el análisis de los datos se utilizó estadística descriptiva, mediante medias y rangos intercuartiles. El análisis de supervivencia se calculó con las curvas de Kaplan-Meier, evaluadas con el programa SPSS ${ }^{\circledR}$ versión 17.0. Para identificar las diferencias se utilizó la prueba Log-rank. Se consideró estadísticamente significativo el valor de $\mathrm{p}<0.05$.

\section{RESULTADOS}

Se registraron 97 pacientes con diagnóstico de cáncer de células renales, a quienes se efectuó nefrectomía parcial o radical; sin embargo, se excluyeron 8 casos por falta de seguimiento y datos incompletos. De esta manera se analizaron 89 pacientes con datos y seguimiento completos. Las características clínicas e histopatológicas se encuentran en el Cuadro 1.

Cuadro 1. Características clínicas e histopatológicas (continúa en la siguiente página)

Variables clínicas

$\mathrm{n}=89(\mathbf{1 0 0} \%)$

Edad (años) media \pm DE

$58.1 \pm 11.26$

Sexo

Masculino

$53(59.6 \%)$

Femenino

$36(40.4 \%)$

Síntomas

Dolor lumbar

$16(18 \%)$

Hematuria

$18(20.2 \%)$

Pérdida de peso

$1(1.1 \%)$

Dos síntomas

$22(24.7 \%)$

$\geq 3$ síntomas

$9(10.1 \%)$

Fortuitos

$23(25.8 \%)$

Tiempo de manifestación de los síntomas

$\leq 30$ días

$19(21.3 \%)$

31 a 90 días

$20(22.5 \%)$

91 a 180 días

11 (12.4)

181 a 360 días

$4(4.5 \%)$

$\geq 360$ días

$12(13.5 \%)$

\section{Comorbilidades}

Diabetes mellitus $24(27 \%)$

Dislipidemia

$10(11.2 \%)$

HAS $^{*+}$

$34(38.2 \%)$

Tabaquismo $40(44.9 \%)$ 
Cuadro 1. Características clínicas e histopatológicas (continuación)

\begin{tabular}{|c|c|}
\hline Variables & $n=89(100 \%)$ \\
\hline \multicolumn{2}{|l|}{ Características tumorales } \\
\hline Tamaño tumoral por TC* $\pm \mathrm{DE}(\mathrm{cm})$ & $7.5 \pm 3.5$ \\
\hline \multicolumn{2}{|l|}{ Riñón afectado } \\
\hline Derecho & $56(62.9 \%)$ \\
\hline Izquierdo & $33(37.1 \%)$ \\
\hline \multicolumn{2}{|l|}{ Localización } \\
\hline Polo superior & $15(16.9 \%)$ \\
\hline Polo medio & $23(25.8 \%)$ \\
\hline Polo inferior & $36(40.4)$ \\
\hline Multicéntrico & $15(16.9 \%)$ \\
\hline \multicolumn{2}{|l|}{ Tipo de intervención } \\
\hline Nefrectomía parcial & $8(9 \%)$ \\
\hline Nefrectomía radical & $81(91 \%)$ \\
\hline \multicolumn{2}{|l|}{ Clasificación histopatológica } \\
\hline T1a & $14(15.7 \%)$ \\
\hline $\mathrm{T} 1 \mathrm{~b}$ & $32(36 \%)$ \\
\hline T2a & $21(23.6 \%)$ \\
\hline $\mathrm{T} 2 \mathrm{~b}$ & $12(13.5 \%)$ \\
\hline T3 & $7(7.9 \%)$ \\
\hline T4 & $3(3.4 \%)$ \\
\hline \multicolumn{2}{|l|}{ Grado nuclear** } \\
\hline Grado I & $8(9 \%)$ \\
\hline Grado II & $53(59.6 \%)$ \\
\hline Grado III & $17(19.1 \%)$ \\
\hline Grado IV & $11(12.4 \%)$ \\
\hline \multicolumn{2}{|l|}{ Estirpe celular } \\
\hline Carcinoma de células claras & $70(78.7 \%)$ \\
\hline Carcinoma papilar & $13(14.6 \%)$ \\
\hline Cromófobo & $6(6.7 \%)$ \\
\hline Tamaño tumoral patológico & $6.7 \pm 3.1$ \\
\hline $\begin{array}{l}\mathrm{TFG}^{+} \text {preoperatoria } \pm \mathrm{DE}(\mathrm{mL} / \\
\mathrm{min} / 1.73 \mathrm{~m} 2)\end{array}$ & $90.3 \pm 24$ \\
\hline $\begin{array}{l}\mathrm{TFG}^{+} \text {posoperatoria } \pm \mathrm{DE}(\mathrm{mL} / \\
\mathrm{min} / 1.73 \mathrm{~m} 2)\end{array}$ & $81.1 \pm 26$ \\
\hline
\end{tabular}

DE: desviación estándar; ${ }^{*} \mathrm{TC}$ : tomografía de abdomen con contraste endovenoso; ${ }^{* *}$ clasificación de Fuhrman; ${ }^{*+h}$ hipertensión arterial sistémica; +tasa de filtración glomerular estimada según fórmula MDRD (Modification of Diet in Renal Disease).

\section{Características clínicas}

La media de edad fue de 58 años (límites de 32 a 84 años), con mayor predominio de hombres (60\%) que mujeres (40\%). El 74\% de los casos manifestó algún síntoma al momento del diagnóstico: hematuria macroscópica 20\%, dolor en el flanco $18 \%$ y ambos síntomas 25\% (Figura 1). Solo 26\% de los casos se diagnosticó de forma incidental. El tiempo de inicio de los síntomas hasta establecer el diagnóstico de tumor renal fue: $21 \%$ en menos de 30 días, $23 \%$ de 30 a 90 días y $30 \%$ más de 90 días (Figura 2). En cuanto a comorbilidades

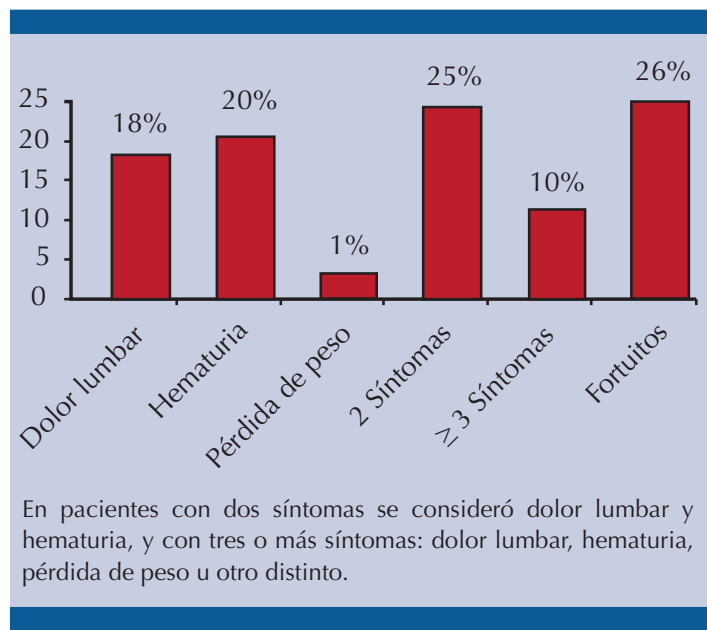

Figura 1. Manifestación clínica del cáncer renal.

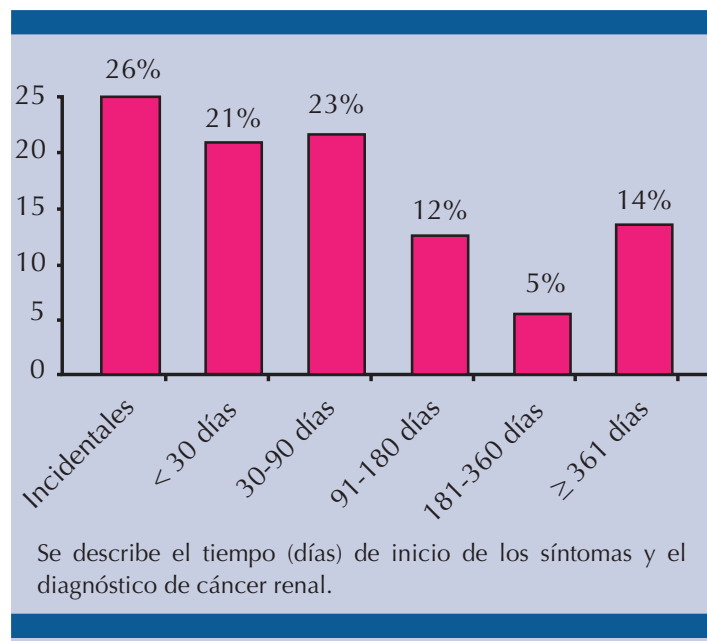

Figura 2. Tiempo de manifestación de los síntomas. 
al momento de la cirugía: 38\% tenía hipertensión, $11 \%$ dislipidemia, $45 \%$ tabaquismo y $27 \%$ diabetes tipo 2. La media de tamaño tumoral estimado por tomografía de abdomen fue de 7.5 $\mathrm{cm}(2.2-20 \mathrm{~cm})$. La media de la tasa de filtrado glomerular prequirúrgica reportó $90.3 \mathrm{~mL} / \mathrm{kg} / \mathrm{m}^{2}$; el $12.3 \%$ tuvo TFG prequirúrgica $\leq 60 \mathrm{~mL} / \mathrm{kg} / \mathrm{m}^{2}$.

\section{Características histopatológicas}

El subtipo histológico predominante fue el de células claras (79\%), seguido del papilar (15\%). El tamaño tumoral promedio fue de $6.7 \mathrm{~cm}$ (DE $\pm 3.14 \mathrm{~cm}$ ). Según la clasificación TNM, 89\% de los casos reportó enfermedad confinada al riñón, principalmente en estadios T1b y T2a, en 36 y $24 \%$ respectivamente (Figura 3 ). El grado de diferenciación celular tipo II, según la clasificación de Fuhrman, predominó en $60 \%$ de los casos. Los tumores localizados en el lado derecho se registraron en $63 \%$ y en el polo inferior en $40 \%$ de los casos.

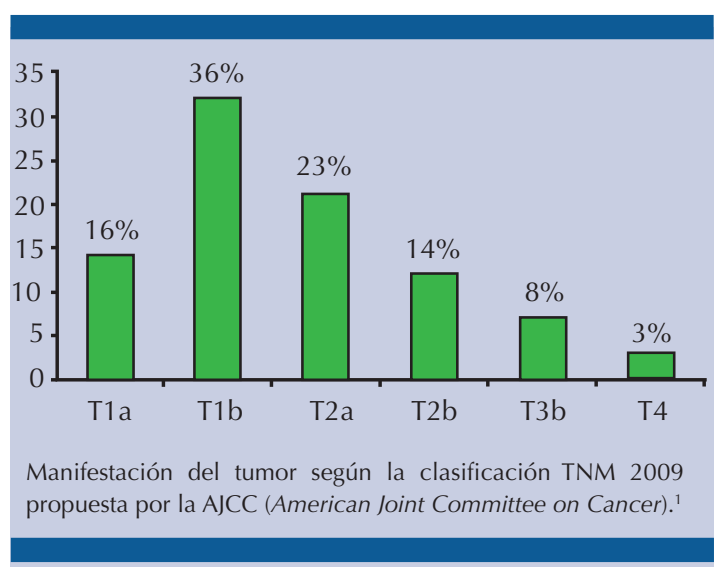

Figura 3. Estadios según la clasificación TNM para cáncer renal.

\section{Resultados quirúrgicos y supervivencia}

La nefrectomía radical abierta se realizó en 91\% versus parcial abierta en $9 \%$ de los pacientes. La media de estancia hospitalaria fue de 4 días
(DE \pm 2.5 días). Respecto a la supervivencia cáncer específica estimada a 5 años para todos los estadios fue de $90 \%$ y supervivencia libre de recurrencia en estadios órgano confinados de $83 \%$. En pacientes con estadio T4, la supervivencia libre de recurrencia a 5 años fue de $33 \%$. La media de seguimiento fue de 26.1 meses (DE \pm 20.1 meses), con límite mínimo de 3 y máximo de 80 meses. La tasa de filtrado glomerular posquirúrgica se calculó en $81.1 \mathrm{~mL} / \mathrm{kg} / \mathrm{m}^{2}$, donde $22 \%$ resultaron con TFG posquirúrgica inmediata de $\leq 60 \mathrm{~mL} / \mathrm{kg} / \mathrm{m}^{2}$ y de éstos, $55 \%$ tenía el mismo valor antes de la cirugía. Al analizar la influencia del tipo de cirugía en la supervivencia cáncer específica no se registraron diferencias estadísticamente significativas $(p=0.33)$.

\section{DISCUSIÓN}

Suárez y sus colaboradores ${ }^{12}$ realizaron un estudio retrospectivo de cohorte, con base en la información del Northern California Kaiser Permanent System, en el que compararon las características del cáncer renal de poblaciones hispanas (principalmente en mexicanos [63\%]) vs caucásicas. Entre sus hallazgos encontraron: edad media al momento del diagnóstico en la población hispana de 59 años, similar a la reportada en nuestro estudio (58 años). Algunas investigaciones han demostrado que diversos factores epidemiológicos aumentan el riesgo de cáncer de células renales, incluido el sexo masculino, enfermedad renal crónica, hipertensión arterial sistémica y sobrepeso u obesidad. ${ }^{13-15}$ De igual forma, Suárez y su grupo ${ }^{12}$ reportaron una prevalencia de diabetes de $24 \%$ en la población con cáncer renal, que sugiere un resultado similar al nuestro. Hollingsworth y su equipo de trabajo ${ }^{16}$ señalaron un aumento en la incidencia de carcinomas renales entre 1983 y 2002, con una tasa de 7.1 a 10.8 casos por cada 100,000 habitantes. Este aumento se registró, principalmente, en pacientes con tumores de 4 $\mathrm{cm}$ o menores. La diferencia entre la población 
mundial y la del norte de México puede explicarse por el contraste de los sistemas de salud a los se encuentran expuestas; sin embargo, se requieren estudios adicionales para determinar este dato. Luciani y su grupo ${ }^{8}$ examinaron más de 1,000 pacientes con diagnóstico de cáncer renal entre 1982 y 1997, con una proporción de diagnóstico fortuito que aumentó de 13 a 59\%. En nuestro estudio, la incidencia fue menor a la reportada en otras series, quizá debido a las limitantes del sistema de salud mexicano, en que las poblaciones vulnerables (áreas suburbanas y rurales) tienen difícil acceso a los estudios de imagen (tomografía computada), que forman parte elemental para establecer el diagnóstico de incidentalomas. ${ }^{2,17} \mathrm{El}$ tratamiento de pacientes con cáncer renal comprende: nefrectomía preservadora de nefronas, nefrectomía radical, vigilancia activa, ${ }^{18}$ crioablación ${ }^{19}$ y radiofrecuencia. ${ }^{20}$ En nuestra serie, todos los pacientes se intervinieron quirúrgicamente. Predominó la nefrectomía radical, lo que coincide con la media de tamaño tumoral de la población (7.5 $\mathrm{cm})$, donde los estadios T1b a T4 se clasificaron en $84 \%$ de los casos, con resultados satisfactorios en cuanto a supervivencia cáncer específica, similares a los reportados en otras series. ${ }^{21}$ En este estudio no se registró la mortalidad transoperatoria ni posoperatoria inmediata. La investigación de MacLennan y su grupo ${ }^{21}$ reportó resultados oncológicos satisfactorios en pacientes con nefrectomía radical y parcial; no reportaron influencia en la evolución ni mortalidad por cáncer de células renales, independientemente del tipo de cirugía, lo que coincide con nuestros hallazgos.

En relación con las características histopatológicas, algunos estudios hispanoamericanos reportan una media de tamaño tumoral de 5 $\mathrm{cm},{ }^{12}$ mientras que nuestra serie señala $7.5 \mathrm{~cm}$, que puede reflejar algunas limitaciones del sistema de salud mexicano para la detección oportuna de tumores renales. Así mismo, Zhang y su equipo ${ }^{22}$ señalan que a mayor grado nuclear mayor correlación con el tamaño tumoral. Sin embargo, en nuestra serie, el grado nuclear con mayor predominio fue el Fuhrman II, tomando en cuenta que nuestra media fue de $7.5 \mathrm{~cm}$. Aunque $9 \%$ de los casos se intervino de nefrectomía parcial, 16\% de los tumores fue menor de $4 \mathrm{~cm}$; por tanto, la diferencia entre el número de nefrectomías parciales en tumores menores de $4 \mathrm{~cm}$ se atribuye al grado de complejidad de las neoplasias, según la nefrometría registrada en la tomografía diagnóstica, y al mismo tiempo influye en la decisión del tipo de cirugía.

El subtipo histológico más frecuente en otras series es el de células claras, ${ }^{9,21}$ similar al de nuestra población (79\%). Los estadios clínicos I/II del AJCC predominaron en $67 \%$ de los casos reportados por Suárez y su grupo. ${ }^{12}$ En nuestra serie, $89 \%$ de los casos correspondió a enfermedad confinada al órgano. De igual forma, el grado de diferenciación celular tipo II predominó en $45 \%$ de los $\operatorname{casos}^{12}$ versus $60 \%$ de nuestro estudio.

La mortalidad por cáncer renal ha disminuido en las últimas décadas, según lo reportado en algunos países europeos. ${ }^{23}$ La supervivencia cáncer específica registrada en esta serie fue similar a la señalada en investigaciones internacionales. ${ }^{12}$

Las limitaciones de este estudio incluyen su naturaleza retrospectiva, la cantidad de pacientes, el seguimiento y la heterogeneidad de la muestra; sin embargo, representa una interesante investigación para reproducirse en otros centros hospitalarios mexicanos, con mayor número de pacientes, con la finalidad de contribuir al registro del cáncer de células renales en México y América Latina.

\section{CONCLUSIÓN}

Las características histopatológicas, el tratamiento y la supervivencia al cáncer de células renales son similares a las reportadas en otros países de 
Europa y Estados Unidos. La manifestación del cáncer renal suele ser sintomática; esto refleja una alta incidencia de estadios avanzados, que sumados a las características del sistema de salud mexicano, se limita la manifestación fortuita del carcinoma de células renales.

\section{Agradecimientos}

Al Dr. Alejandro García Ramírez por su invaluable apoyo y enseñanza.

\section{Conflicto de interés}

Los autores declaran no tener conflicto de interés.

\section{Financiamientos}

La elaboración de este trabajo no recibió financiamiento.

\section{REFERENCIAS}

1. Ljungberg B, Bensalah K, Canfield S, Dabestani S, et al. EAU Guidelines on Renal Cell Carcinoma: 2014 Update. Eur J. 2015;67:913-924.

2. Patard JJ. Incidental renal tumouinrs. Curr Opin Urol. 2009;19(5):454-458. doi:10.1097/MOU.0b013e32832f0ccd.

3. Adibi M, Karam JA, Wood CG. Reporting geographic and temporal trends in renal cell carcinoma: Why is this important? Eur Urol. 2015;67(3):531-532. doi:10.1016/j. eururo.2014.10.030.

4. Siegel RL, Miller KD, Jemal A. Cancer Statistics, 2017. CA Cancer J Clin 2017;67(1):7-30. doi:10.3322/caac.21387.

5. Thompson RH, Hill J, Babayev Y, Cronin A, et al. Risk of metastatic renal cell carcinoma according to tumor size. J Urol 2010;182(1):41-45. doi:10.1016/j.juro.2009.02.128.RISK.

6. Lorente D, Trilla E, Meseguer A, Planas J, et al. Revisión sistemática de los factores pronósticos del carcinoma renal. Actas Urol Esp 2017;41(4)215-25.

7. Eggener SE, Yossepowitch O, Pettus JA, et al. Renal cell carcinoma recurrence after nephrectomy for localized disease: predicting survival from time of recurrence. J Clin Oncol 2006;24:3101-3106.

8. Luciani LG, Cestari R, Tallarigo C. Incidental renal cell carcinoma-age and stage characterization and clinical implications: study of 1092 patients (1982-1997). Urology. 2000;56(1):58-65.
9. DeCastro GJ, McKiernan JM. Epidemiology, clinical staging, and presentation of renal cell carcinoma. Urol Clin North Am 2008;35(4):581-592. doi:10.1016/j.ucl.2008.07.005.

10. National Cancer Institute. Cancer Stat Fact: Kidney and Renal Pelvis Cancer. [en línea]. Dirección URL <https://seer. cancer.gov/statfacts/html/kidrp.html>.

11. Levey AS, Coresh J, Greene T, Stevens LA, et al. Using standardized serum creatinine values in the modification of diet in renal disease study equation for estimating glomerular filtration rate. Ann Intern Med 2006;145(4):247-254. doi:10.7326/0003-4819-145-4-200608150-00004.

12. Suarez-Sarmiento A, Yao X, Hofmann JN, Syed JS, et al. Ethnic disparities in renal cell carcinoma: An analysis of Hispanic patients in a single-payer healthcare system. Int J Urol 2017;24(10):765-770. doi:10.1111/iju.13424.

13. Chow WH, Dong LM, Devesa SS. Epidemiology and risk factors for kidney cancer. Nat Rev Urol 2010;7:245.

14. Colt JS, Schwartz K, Graubard BI, Davis F, R, et al. Hypertension and risk of renal cell carcinoma among white and black Americans. Epidemiol 2011;22:797.

15. Purdue MP, Moore LE, Merino MJ, Boffetta P, et al. An investigation of risk factors for renal cell carcinoma by histologic subtype in two case-control studies. Int J Cancer 2013;132:2640

16. Hollingsworth JM, Miller DC, Daignault S, Hollenbeck BK Rising incidence of small renal masses: A need to reassess treatment effect. J Natl Cancer Inst. 2006;98(18):13311334. doi:10.1093/jnci/djj362.

17. Sand KE, Hjelle KM, Rogde ÅJ, Gudbrandsđottir G, Bostad L, Beisland C. Incidentally detected renal cell carcinomas are highly associated with comorbidity and mortality unrelated to renal cell carcinoma. Scand J Urol 2013;47(6):462-471. doi:10.3109/21681805.2013.777364.

18. Smaldone MC, Corcoran AT, Uzzo RG. Active surveillance of small renal masses. Nat Rev Urol 2013;10(5):266-274. doi:10.1038/nrurol.2013.62.

19. Abouassaly R, Lane BR, Novick AC. Active surveillance of renal masses in elderly patients. J Urol 2008;180:505-509. doi:10.1016/j.juro.2008.04.033.

20. Levinson AW, Su LM, Agarwal D, Sroka M, et al. Long-term oncological and overall outcomes of percutaneous radio frequency ablation in high risk surgical patients with a solitary small renal mass. J Urol. 2008;180(2):499-504. doi:10.1016/j.juro.2008.04.031.

21. MacLennan S, Imamura M, Lapitan MC, Omar MI, et al. Systematic review of oncological outcomes following surgical management of localised renal cancer. Eur Urol 2012;61:972-993. doi:10.1016/j.eururo.2012.02.039.

22. Zhang C, Li X, Hao H, Yu W, He Z, Zhou L. The correlation between size of renal cell carcinoma and its histopathological characteristics: A single center study of 1867 renal cell carcinoma cases. BJU Int 2012;110(11 B):481-485. doi:10.1111/j.1464-410X.2012.11173.x.

23. Sun M, Shariat SF, Cheng C, Ficarra V, Murai M, Oudard $S$, et al. Prognostic factors and predictive models in renal cell carcinoma: A contemporary review. Eur Urol 2011;60(4):644-661. doi:10.1016/j.eururo.2011.06.041. 